\title{
Chromosomes : étonnants polymères !
}

Maria Barbi ${ }^{(1)}$, Annick Lesne ${ }^{(1,2)}$, Julien Mozziconacci ${ }^{(1)}$

et Jean Marc Victor ${ }^{(1)}$ (victor@lptmc.jussieu.fr)

(1) Équipe Modélisation Multi-échelles de la Matière Vivante,

LPTMC, CNRS UMR 7600, UMPC, 4 place Jussieu, 75252 Paris Cedex 05

(2) Institut de Génétique Moléculaire de Montpellier (Université de Montpellier/CNRS),

1919 route de Mende, 34293 Montpellier Cedex 5.

La molécule d'ADN est le support

physique de l'information génétique.

Ce long polymère est compacté

grâce à des protéines pour former

les chromosomes. Ce repliement

assure la régulation de l'expression

des gènes au cours de la vie

des cellules.

La biochimie des chromosomes est

un sujet d'intenses investigations,

mais il apparait aujourd'hui

que les propriétés physiques

des chromosomes sont aussi

au cœur de leur fonctionnement.

Ces propriétés peuvent être

décrites en combinant physique

statistique et physique

des polymères en solution.

Dans cet article, nous présentons

les méthodes multi-échelles

que nous avons développées

pour reconstruire et animer

l'architecture fonctionnelle

des chromosomes.

Les mots suivis d'un astérisque sont définis dans le glossaire, p. 14
L'ADN est souvent considéré comme la "molécule de la vie ", depuis que Watson et Crick en ont découvert la structure en double hélice en 1953. Tout organisme vivant possède en effet, nichées dans chacune de ses cellules, une ou plusieurs doubles hélices d'ADN qui, associées à des protéines par des liaisons électrostatiques (liaisons hydrogène ou liaisons ioniques), constituent les chromosomes de l'organisme. Chacun des deux brins d'une double hélice d'ADN est un polymère réalisé par l'enchaînement de quatre types de monomères appelés nucléotides ${ }^{\star}$, repérés respectivement par une des quatre lettres : A, T, G, C. La séquence de lettres présente sur un brin, équivalente à celle du brin complémentaire, encode les protéines que doit synthétiser l'organisme. Mais la double hélice est aussi un polymère, dont les monomères sont les paires de nucléotides complémentaires - on parle d'habitude de "paires de bases ": A étant toujours appariée avec T, G toujours avec C. La longueur d'un chromosome varie d'une espèce à l'autre de plusieurs ordres de grandeur : chez la levure de bière, l'un des plus petits organismes eucaryotes ${ }^{(a)}$ (c'est d'ailleurs un unicellulaire), les tailles chromosomiques sont de l'ordre de la mégabase (Mb, un million de paires de bases), chez la mouche drosophile de la dizaine de $\mathrm{Mb}$ et chez l'homme de la centaine de $\mathrm{Mb}$. Les chromosomes sont ainsi, de très loin, les plus longues molécules connues, pouvant atteindre plusieurs dizaines de centimètres pour les plus longs $^{(b)}$. Or les chromosomes doivent tenir dans le noyau d'une cellule, dont le diamètre ne dépasse pas la dizaine de microns ; et encore, chez l'homme, ce sont 46 chromosomes $^{(\mathrm{c})}$ qui doivent tenir dans un même noyau ! Ce tour de force est réalisé par différents niveaux de repliement conduisant à une organisation hiérarchique du chromosome (fig. 1). De cette organisation, on ne connaît précisément que le tout premier niveau de compaction : le nucléosome, obtenu par l'enroulement d'environ 200 paires de bases de la double hélice d'ADN autour d'une bobine nanométrique composée de protéines (fig. 1 ; voir aussi l'encadré, p. 12). Le chapelet de nucléosomes ainsi obtenu est encore un polymère, environ dix fois plus court que la double hélice d'ADN. Lorsque l'enchaînement des nucléosomes est régulier - la longueur d'ADN entre deux nucléosomes est alors constante - ce polymère peut à son tour s'organiser en fibre de chromatine (fig. 1 ; voir aussi l'encadré).

\section{Une organisation fonctionnelle}

La structure et la dynamique de ce polymère-chromosome font l'objet d'intenses recherches et de débats enflammés. Car si la double hélice d'ADN révélée par Watson et Crick fournissait immédiatement la solution au problème de la reproduction du matériel génétique et avait donc une importance considérable pour la biologie, c'est encore loin d'être le cas pour le chapelet de nucléosomes dont les propriétés physiques n'aident guère à comprendre les fonctions biologiques remplies par les chromosomes. Et pourtant, l'organisation des chromosomes dans l'espace du noyau cellulaire recèle probablement une part des secrets du fonctionnement des organismes pluricellulaires. Chez ces organismes, en effet, toutes les cellules possèdent le même 
Dans une micromanipulation par pinces magnétiques, une extrémité d'une molécule unique d'ADN est accrochée à une surface et l'autre extrémité à une bille magnétique soumise à une force connue et qu'on peut faire tourner de manière contrôlée ; on mesure alors la distance de la bille à la surface, donc l'extension de la molécule, en fonction de la force et/ou du nombre de tours appliqués (fig. E1).

Les micromanipulations par pinces magnétiques permettent de recueillir des informations globales sur la molécule d'ADN : son extension moyenne et la force qui lui est appliquée. Mais le détail des conformations de la molécule reste inaccessible. Nous avons donc développé un outil de simulation gros-grain de la double hélice d'ADN, qui permet de la modéliser par une chaîne de cylindres articulés au moyen de liaisons rotule auxquelles sont associées des forces et couples de rappel qui correspondent respectivement aux rigidités en flexion et en torsion du filament d'ADN (encart de la figure E1). Un autre intérêt majeur de ce type de simulation est le calcul des forces et surtout des couples exercés sur l'ADN - ces derniers étant difficilement mesurables expérimentalement, notamment dans des situations où c'est le nombre de tours qui est imposé à la bille. On peut également explorer des conditions un peu différentes des conditions expérimentalement accessibles, par exemple en imposant un couple à la bille et en mesurant le nombre de tours qu'elle fait sur elle-même [4].

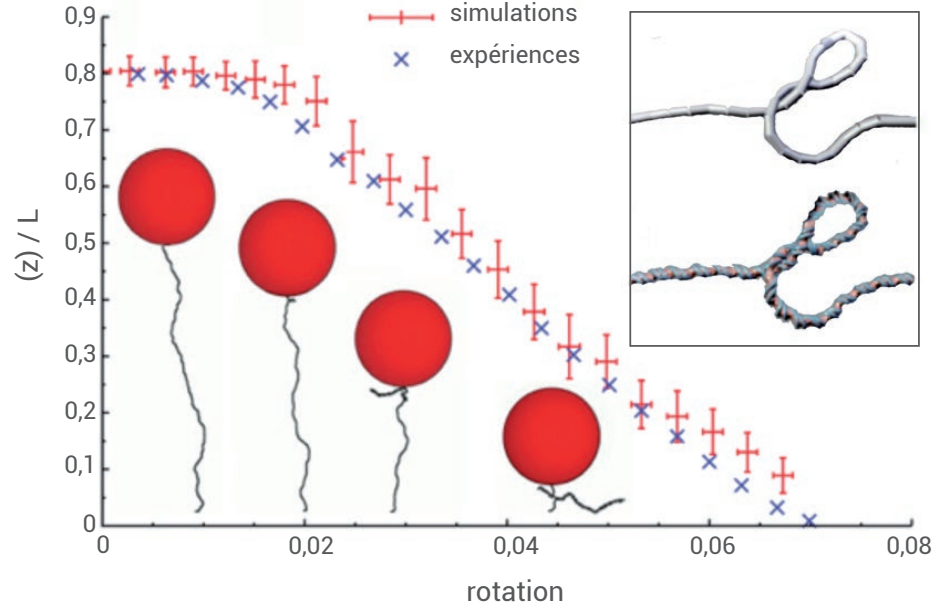

E1. Comparaison d'une courbe de rotation-extension expérimentale obtenue par pinces magnétiques [Mosconi et al., PRL 102 (2009) 078301] et de sa reproduction par simulation numérique. L'ADN (encart, bas) est modélisé à l'aide de l'outil de simulation macromoléculaire CGMDODE ${ }^{(1)}$ comme une chaîne de cylindres articulés (encart, haut) : la taille des cylindres (ici 10 bases, $3,4 \mathrm{~nm}$ ) est inférieure aux longueurs de persistance de l'ADN, et des couples de force sont appliqués au niveau des articulations pour reproduire les propriétés élastiques de l'ADN. Sous les points de mesure, des instantanés de la simulation montrent comment la rotation appliquée à la bille (en rouge) induit la formation de structures surenroulées, appelées plectonèmes, qui « absorbent » la rotation en réduisant la longueur $\langle z>$ de la molécule proportionnellement à la rotation appliquée. La rotation est ici une mesure relative de la torsion par rapport à la torsion intrinsèque de la double hélice.

(1) Coarse-Grained Macromolecular Dynamics with Open Dynamics Engine.

\section{La fibre de chromatine, un ressort accordable}

Pour étendre ces résultats au cas de la chromatine, il est nécessaire de prendre en compte la structure des complexes nucléoprotéiques élémentaires qui la composent - les nucléosomes - et la manière dont ils organisent l'ADN dans l'espace. Leur disposition régulière le long de la molécule d'ADN permet en effet de la " plier » et de l'organiser dans une architecture qui la fait ressembler à un ressort hélicoïdal aux spires complexes, dont le fil est la double hélice d'ADN. Nous avons développé un modèle gros-grain de la structure du nucléosome en nous appuyant sur une analyse en modes normaux de sa structure moléculaire, qui permet d'en identifier les zones flexibles et de reconstituer le complexe en reliant entre eux de manière appropriée une douzaine de corps rigides (fig. E2). Ainsi, il a été possible de simuler le comportement d'une fibre de chromatine soumise à des contraintes mécaniques, et notamment de calculer les efforts mécaniques (forces, couples) induits par une manipulation en torsion et en traction par des pinces magnétiques.

E2. Modélisation d'un nucléosome (a) et d'un segment de chromatine avec 10 nucléosomes (b) par CGMDODE. Le nucléosome est modélisé à partir d'une analyse en modes normaux qui, en identifiant les points charnière de la structure, permet de la décomposer en une série de corps rigides articulés opportunément. Dans chaque nucléosome, les cylindres qui représentent I'ADN s'enroulent ainsi autour d'une structure centrale qui représente les protéines histones, ici coloriées comme les segments d'ADN auxquels elles sont liées.

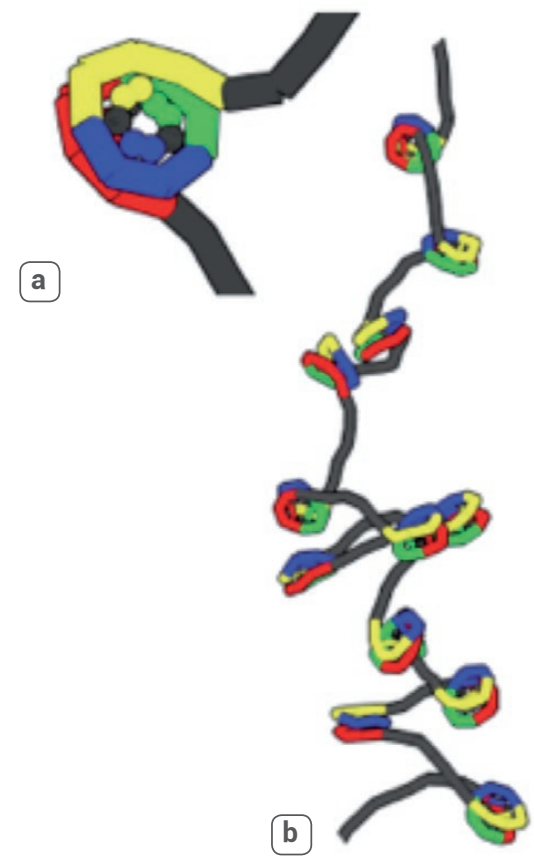



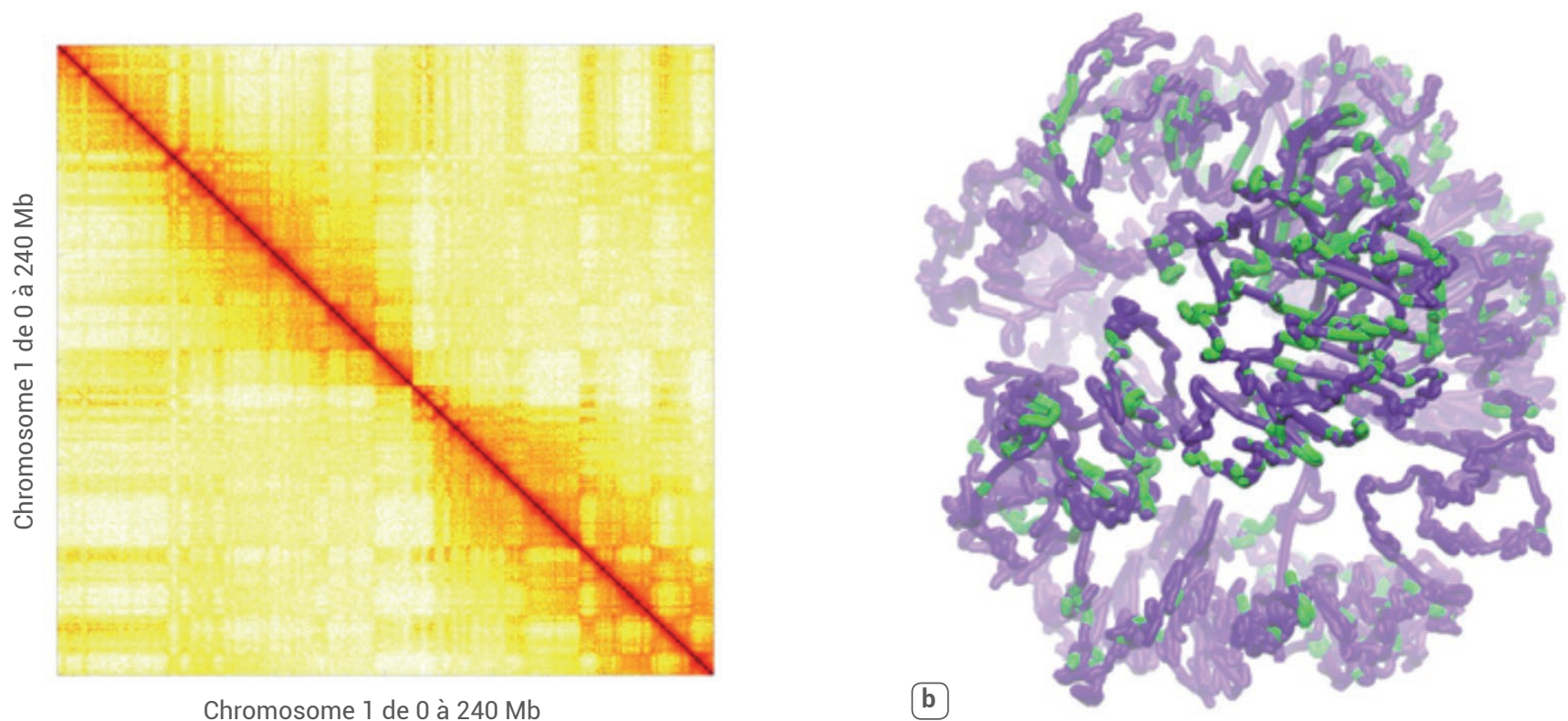

2. Reconstruction du chromosome 1 humain.

(a) Matrice de contacts, à la résolution de $30 \mathrm{~kb}$. Les coordonnées le long de l'axe horizontal (ou vertical, ce sont les mêmes) indiquent la position le long du génome, en suivant l'ADN ; la couleur graduelle des pixels varie avec le nombre de contacts, d'autant plus foncée que ce nombre est élevé. On remarque une zone rouge foncé autour de la diagonale, indiquant que les sites voisins, à l'échelle de quelques centaines de kb, sont davantage en contact que des régions plus distantes le long du génome.

(b) Structure 3D du chromosome 1 humain reconstruite à partir de (a), où sont figurés en vert les gènes transcrits et en violet la chromatine condensée (comme sur la figure 1).

\section{\>}

contraintes physiques qui, aux différents niveaux d'organisation de l'ADN au sein des chromosomes, limitent ou orientent les fluctuations conformationnelles résultant de l'agitation thermique. Ils donnent donc des informations importantes, mais indirectes, sur l'architecture du noyau. Car comment passer des contacts à la structure 3D du polymère-chromosome?

L'algorithme que nous avons développé pour relever ce défi est fondé sur des résultats mathématiques (positionnement multidimensionnel), donnant les coordonnées tridimensionnelles d'un ensemble de points à partir de la connaissance de toutes leurs distances mutuelles. Lorsque les distances sont entachées d'erreurs expérimentales, la méthode donne l'approximation optimale de ces coordonnées. Pour appliquer cette méthode, l'idée a été de construire un réseau virtuel, dont les nœuds sont les sites génomiques. Dans cette représentation, deux sites seront connectés par un lien direct s'ils ont établi un contact. Pour tirer parti du caractère quantitatif des données, nous avons attribué à chaque lien une longueur, inversement proportionnelle au nombre de contacts entre les sites qu'il connecte. On peut ensuite calculer une distance abstraite entre deux sites quelconques comme la longueur du plus court chemin reliant les sites sur le réseau (souvent inférieure à la longueur $\mathrm{du}$ lien direct). Cette notion satisfait les propriétés mathématiques requises pour définir une vraie distance (en particulier, elle est symétrique et vérifie l'inégalité triangulaire). Elle ne reflète qu'indirectement la distance géométrique entre deux sites génomiques mais, et c'est là l'avancée essentielle, elle est définie pour toute paire de sites et peut ainsi servir de base à la reconstruction des coordonnées 3D suivant la méthode mathématique mentionnée ci-dessus.

L'algorithme, baptisé ShRec3D pour 3D Shortest-path Reconstruction, a été validé sur des données simulées (simulation du noyau de levure, voir plus loin la figure 4b), en comparant la structure générée dans la simulation et l'image reconstruite à partir des contacts observés dans cette structure. Puis nous l'avons mis en œuvre sur des matrices de contacts réelles, en particulier humaines. Une des forces de cet algorithme est la rapidité de son temps d'exécution : il permet ainsi d'exploiter dans un temps de calcul raisonnable (quelques minutes pour un millier de sites génomiques) l'intégralité des données expérimentales, à la résolution la plus fine [2].

Cette reconstruction (fig. 2b) permet de visualiser l'organisation hiérarchique des chromosomes aux grandes échelles, précisant le schéma de la figure 1 au-delà de la fibre de chromatine. On peut ainsi décrire : (i) des boucles de chromatine (de quelques dizaines de $\mathrm{kb}$ ), et (ii) à un niveau supérieur d'organisation, une structuration le long du génome en domaines de quelques centaines de $\mathrm{kb}$, tendant à occuper des régions distinctes dans l'espace 3D du noyau, et appelés pour cette raison des domaines topologiques. Ces caractéristiques changent avec le type cellulaire (neurones, cellules de foie, de muscle, etc.) : leur visualisation donne une intuition immédiate des variations de l'organisation 3D du génome et guide l'étude de leurs possibles rôles dans la différenciation cellulaire.

La reconstruction présentée ici fournit une image moyenne, puisqu'elle est obtenue sur un ensemble de conformations (une conformation par cellule). Cette image moyenne est d'autant plus fidèle que l'échelle est plus grande. De nouvelles techniques de "capture de conformations chromosomiques » sur cellules uniques commencent à voir le jour, pour lesquelles l'algorithme $\operatorname{ShRec} 3 D$ fournit des images fidèles. L'application de cet algorithme aux données expérimentales montre une grande variabilité dans les conformations des chromosomes. Cette variabilité d'une 


\section{Glossaire}

Blob : représentation schématique d'un segment de polymère sous forme de bille, dont le diamètre est égal à la longueur de corrélation des fluctuations de concentration des monomères. À l'intérieur d'un blob, les monomères interagissent (par répulsion stérique essentiellement), tandis que les monomères de deux blobs différents n'ont pas d'interaction : les polymères se comportent donc comme des marches aléatoires de blobs. Dans une brosse, les chaînes tendent à s'aligner, comme les poils d'un tapis dru, et ne s'enchevêtrent pas.

Centromère : les deux copies d'un chromosome obtenues après réplication du matériel génétique commencent par rester accrochées au niveau d'une région du génome, appelée centromère (les deux copies, appelées chromatides sœurs, forment alors un X).

Épigénétique : se dit d'une modification chimique qui affecte une base de l'ADN (pour l'essentiel méthylation, c'est-à-dire ajout d'un groupement méthyle) ou les histones (méthylation, acétylation, etc.), mais qui ne change pas la séquence génomique.

Expression génétique : un gène est exprimé quand sa séquence d'ADN est transcrite en ARN messager (en réponse à un message biochimique). Il est réprimé si des modifications épigénétiques empêchent sa transcription.

Histones : protéines portant une forte charge positive ; chaque nucléosome est un assemblage de huit histones, stabilisé par la charge négative de la double hélice d'ADN.

kb : kilobase, c'est-à-dire 1000 paires de bases.

Nucléotides : un nucléotide est une molécule composée d'un sucre (pentose), d'un groupement phosphate et d'une base particulière. On repère chaque nucléotide d'une séquence d'ADN par l'initiale de la base qu'il porte : A (Adénine), T (Thymine), G (Guanine), C (Cytosine).

Site génomique : séquence d'ADN située à un endroit unique et invariable du génome. Sa longueur dépend du contexte biologique et/ou expérimental, allant de quelques paires de base pour un site de liaison d'une protéine jusqu'à plusieurs kb lorsque la résolution expérimentale ne permet pas de déterminer plus finement la localisation.
>>

cellule à l'autre suggère que le chromosome est un objet dynamique présentant de larges fluctuations. L'origine physique de ces fluctuations n'est cependant pas claire : s'agit-il simplement de fluctuations thermiques autour de l'équilibre ? Ou bien des processus actifs sont-ils en cause ? Pour répondre à ces questions, des mesures dynamiques sont nécessaires, comme discuté ci-dessous.

\section{La levure de bière : une brosse de polymères}

Un organisme de choix pour observer la dynamique des chromosomes est la levure de bière. Cet organisme unicellulaire a un génome presque mille fois plus petit que le génome humain (seulement $13 \mathrm{Mb}$ au total) et très peu de marques épigénétiques. Cette simplification laisse espérer que la physique des polymères puisse être un guide pertinent dans ce cas.

$\mathrm{La}$ disposition particulière des seize chromosomes de la levure de bière, accrochés en permanence à la membrane nucléaire chacun par son centromère* (fig. 3a), suggère que leur organisation dans l'espace pourrait être celle d'une brosse de polymères à l'équilibre thermodynamique (fig. 3b). Une collaboration avec trois

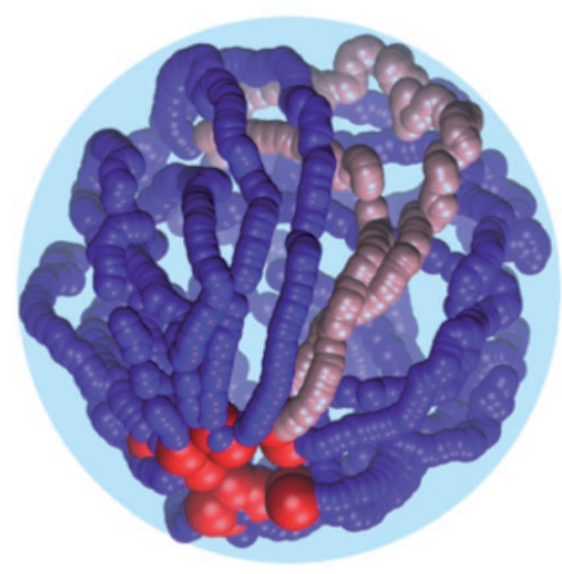

a

b

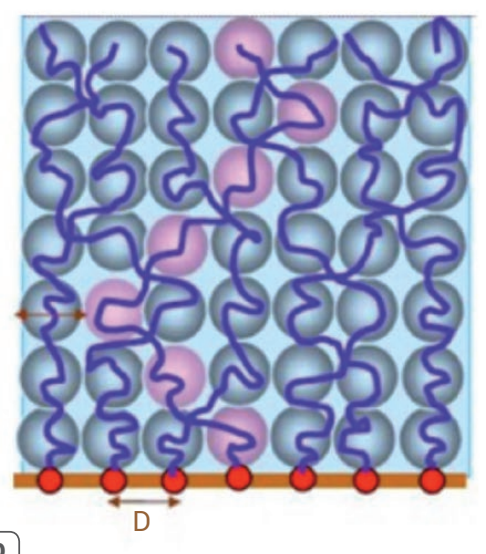

\section{Brosse de polymères.}

(a) Représentation schématique d'un noyau de levure de bière. Les chromosomes sont représentés par des chaînes de billes (en bleu). Chaque chromosome est attaché à la membrane nucléaire par son centromère* (en rouge). Tous les centromères sont attachés ensemble.

(b) Schéma (coupe) d'une brosse de polymères. Tous les polymères (chaînes en bleu) sont greffés sur une surface à intervalles réguliers (la distance $D$ entre polymères adjacents est aussi le diamètre des blobs*). Les blobs sont figurés par les billes bleues transparentes. La trajectoire d'une chaîne en particulier, dont les blobs sont surlignés en rose, montre qu'elle ne revient pas en arrière vers la surface de greffage (" polymère dirigé »), mais qu'elle effectue une marche aléatoire dans les directions transverses. 


\section{Un polymère-chromosome fonctionnel}

Ainsi, il est très étonnant que l'organisation des chromosomes dans un noyau de levure soit essentiellement fixée par la physique classique des polymères. Or, l'organisation nucléaire, impliquée chez les organismes pluricellulaires dans des processus aussi mystérieux que la différenciation cellulaire et le développement embryonnaire, est aussi à l'œuvre tout au long de la vie de la cellule dans les grandes fonctions que sont la régulation de l'expression des gènes, la réparation des cassures de l'ADN, la réplication du génome et l'ensemble des mécanismes qui assurent la division cellulaire (en particulier la bonne ségrégation des chromosomes dans les deux cellules filles). Ces phénomènes complexes concernent aussi la levure de bière. On peut donc espérer que la physique des polymères adaptée pour traiter des objets aussi complexes que les polymères-chromosomes décrits dans cet article - soit aussi pertinente pour les organismes pluricellulaires. C'est autour de cette question que nous continuons à développer notre recherche. Nous ne sommes pas seuls dans cette aventure : nous interagissons avec un grand nombre d'équipes françaises expérimentales et théoriques, en biologie, physique et médecine, grâce au "GdR ADN ", réseau de recherche thématique interdisciplinaire du CNRS consacré à l'architecture et la dynamique nucléaires (http://gdradn.lptmc.jussieu.fr), que nous avons lancé en 2012. Le problème est loin d'être résolu mais, si la route est encore longue, l'enthousiasme est partagé ! -

(a) Les organismes eucaryotes comprennent en particulier les animaux, les plantes et les champignons. Leurs cellules ont un noyau qui contient les chromosomes.

(b) Chaque paire de base a une longueur de 0,34 nm selon l'axe de la double hélice, donc $100 \mathrm{Mb}$ d'ADN correspondent à une longueur de $3,4 \mathrm{~cm}$ en suivant la molécule d'ADN

(c) 22 paires d'autosomes (chromosomes non sexuels) plus deux chromosomes sexuels.

(d) C'est seulement au cours de la division cellulaire que les chromosomes s'individualisent et apparaissent sous forme de bâtonnets, comme dans les caryotypes.

Nous remercions nos collaborateurs passés et présents sur ces sujets de recherche, en particulier Aurélien Bancaud, Bertrand Caré, Pascal Carrivain, Ruggero Cortini, Christophe Lavelle, Hua Wong.
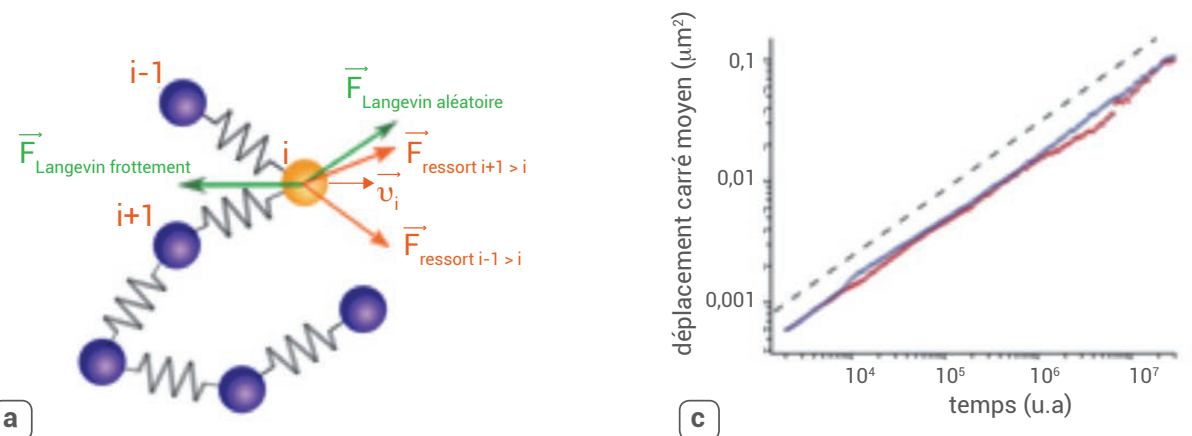

c

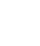

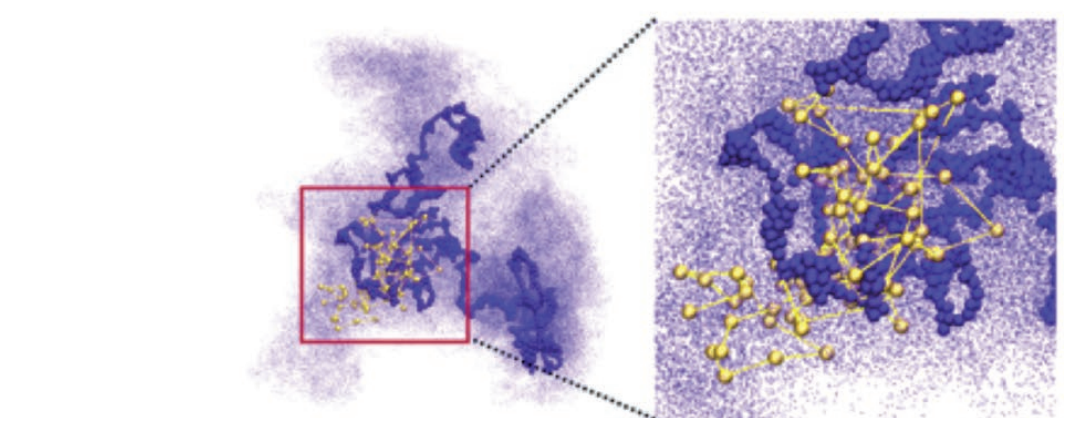

b

4. Dynamique d'un chromosome de levure de bière.

(a) Représentation schématique du modèle bille-ressort (aussi appelé modèle de Rouse). Chaque bille est soumise à la force des ressorts et aux forces de Langevin (friction visqueuse et chocs aléatoires).

(b) Instantané de simulation d'un chromosome de levure, dont le noyau peut être simulé comme un ensemble de chaînes bille-ressort ancrées à la membrane nucléaire, chacune par son milieu et évoluant dans un volume sphérique sous l'effet de l'agitation thermique. Les lignes bleues correspondent, pour une chaîne donnée, à un échantillon de configurations prises à des instants différents. Une de ces configurations est tracée en ligne épaisse, agrandie à droite. Lorsqu'un monomère est marqué comme dans les expériences (point en jaune), on peut suivre son mouvement erratique au cours du temps.

(c) Déplacement carré moyen d'un monomère. Le mouvement d'un monomère est caractérisé par son déplacement carré moyen (mean square displacement ou MSD). Aussi bien dans les expériences que dans les simulations on observe que le MSD augmente comme $t^{1 / 2}$ sur plusieurs décades, au lieu de $t$ comme ce serait le cas pour une diffusion normale. Le déplacement quadratique moyen (root-mean-square displacement ou RMSD), égal à la racine carrée du MSD, est une mesure de la taille du domaine exploré par le site génomique : il est ici comparable à la taille du noyau (environ $400 \mathrm{~nm}$ pour les plus longues trajectoires enregistrées).

\section{Pour en savoir plus}

1· R. Cortini et al., "The physics of epigenetics", Reviews of Modern Physics 88 (2016) 025002.

2• A. Lesne et al., "Reconstructing 3D genomes from chromosomal contact maps", Nature Methods 11 (2014) 1141-1143.

Voir également la revue de presse www.cnrs.fr/inp/spip.php?article3225 .

3• H. Hajjoul et al., "High-throughput chromatin motion tracking in living yeast reveals the flexibility of the fiber throughout the genome", Genome Research 23 (2013) 1829-1838.

4• P. Carrivain, M. Barbi et J.M. Victor, "In silico single-molecule manipulation with rigid body dynamics: an efficient tool", PLOS Computational Biology 10 (2013) e1003456.

Voir également la revue de presse www.cnrs.fr/inp/spip.php?article2645 .
- A. Bancaud et al., "Torsional plasticity of single chromatin fibers revealed by torsional manipulation", Nat. Struc. Mol. Biol. 13 (2006) 444-450.

-A. Boettiger et al., "Super-resolution imaging reveals distinct chromatin folding for different epigenetic states", Nature 529 (2016) 418-422.

- L. Lazar-Stefanita et al., "Cohesins and condensins orchestrate the $4 \mathrm{D}$ dynamics of yeast chromosomes during the cell cycle", EMBO J. 36 (2017) 2684-2697. www.ncbi.nlm.nih.gov/pubmed/28729434

- S. Wang et al., "Spatial organization of chromatin domains and compartments in single chromosomes", Science 353 (2016) 598-602. 